\title{
Density Functional Investigation of Graphene Doped with Amine-Based Organic Molecules
}

\author{
Yeun Hee Hwang, ${ }^{1}$ Hyang Sook Chun, ${ }^{2}$ Kang Min Ok, ${ }^{1}$ \\ Kyung-Koo Lee, ${ }^{3}$ and Kyungwon Kwak ${ }^{1}$ \\ ${ }^{1}$ Department of Chemistry, Chung-Ang University, 221 Heukseok-dong, Dongjak-gu, Seoul 156-756, Republic of Korea \\ ${ }^{2}$ School of Food Science and Technology, Chung-Ang University, Ansung, Gyeonggi 456-756, Republic of Korea \\ ${ }^{3}$ Department of Chemistry, Kunsan National University, Gunsan, Jeollabuk-do 573-701, Republic of Korea
}

Correspondence should be addressed to Kyung-Koo Lee; kklee@kunsan.ac.kr and Kyungwon Kwak; kkwak@cau.ac.kr

Received 7 June 2015; Accepted 2 July 2015

Academic Editor: Jin W. Seo

Copyright (C) 2015 Yeun Hee Hwang et al. This is an open access article distributed under the Creative Commons Attribution License, which permits unrestricted use, distribution, and reproduction in any medium, provided the original work is properly cited.

To improve the electronic properties of graphene, many doping techniques have been studied. Herein, we investigate the electronic and molecular structure of doped graphene using density functional theory, and we report the effects of amine-based benzene dopants adsorbed on graphene. Density functional theory (DFT) calculations were performed to determine the role of amine-based aromatic compounds in graphene doping. These organic molecules bind to graphene through long-range interactions such as $\pi-\pi$ interactions and $\mathrm{C}-\mathrm{H} \cdots \pi$ hydrogen bonding. We compared the electronic structures of pristine graphene and doped graphene to understand the electronic structure of doped graphene at the molecular level. Also, work functions of doped graphene were obtained from electrostatic potential calculations. A decrease in the work function was observed when the amine-based organic compounds were adsorbed onto graphene. Because these systems are based on physisorption, there was no obvious band structure change at point $K$ at the Fermi level after doping. However, the amine-based organic dopants did change the absolute Fermi energy levels. In this study, we showed that the Fermi levels of the doped graphene were affected by the HOMO energy level of the dopants and by the intermolecular charge transfer between the adsorbed molecules and graphene.

\section{Introduction}

Graphene, a two-dimensional hexagonal lattice of carbon, has recently attracted much interest as a flexible and transparent conductive electrode due to its high conductivity [1-3] and low optical absorption [3-5]. By controlling the graphene work function, the conductivity can be improved through reducing the contact barriers between graphene and an electrode device [6]. The conductivity can be tuned by doping techniques such as substitution doping [7], chemical doping [8], and spontaneous surface charge transfer between adsorbed materials and carbon atoms [9]. Previous studies have shown that derivatives of benzene can bind to graphene sheets because of the formation of strong $\pi-\pi$ interactions between the aromatic ring and the extended $\pi$-system of graphene. Also, the degree of doping of graphene can be adjusted by modulating the electron-donating abilities of the benzene derivatives by adding electron-donating or electron-withdrawing group substituents. Electron-donating groups increase the electron density on aromatic molecules; thus, they lead to n-type doping of graphene. For example, 1,5-naphthalenediamine is an aromatic molecule with an electron-donating amine functional group $\left(-\mathrm{NH}_{2}\right)$. In contrast, aromatic molecules with electron-withdrawing groups, for example, tetrasodium 1,3,6,8-pyrenetetrasulfonic acid (TPA), cause p-type doping of graphene because these substituents decrease the electron density on the aromatic ring system of the organic dopant [10]. In addition, aminebased organic compounds are known to be efficient n-type dopants because amine $\left(-\mathrm{NH}_{2}\right)$ functional groups have lone pairs, which can donate electrons to the graphene sheet $[11,12]$. Density functional theory (DFT) calculations can be used to obtain detailed information about the bond types and electronic structure of doped graphene, enabling the examination and analysis of these systems [13]. Recently, DFT calculations were used to show that both pristine graphene 
and Al-doped graphene are potential candidates for the detection of the toxic compound acrolein $\left(\mathrm{C}_{3} \mathrm{H}_{4} \mathrm{O}\right)$ [14].

In this study, we used amine-based benzene derivatives to investigate the effect of amine-based organic molecules on the electronic and molecular structure of graphene. The properties of the organic molecules were modulated by the addition of functional groups such as phenyl rings (1), butylphenyl groups (2), and acetylphenyl groups (3). To investigate the role of amine derivatives in graphene doping, we carried out DFT calculations. Geometry optimizations were used to find stable equilibrium geometries, and electrostatic potential calculations were performed to obtain the work functions of both pristine graphene sheet and graphene doped with amine-based dopants. In addition, the electronic structure of pristine graphene and that of doped graphene were compared to understand and improve the performance of graphenebased electrode devices at the molecular level.

\section{Computational Methods}

2.1. Geometry Optimization. All DFT calculations were performed with the $\mathrm{DMol}^{3}$ software package [15] to study the interactions between graphene and the amine-based organic molecules and to predict the electronic structure change of graphene when the organic molecules are physisorbed onto graphene. To mimic the extended structure, we used a periodic boundary condition (PBC). This was used for both the pristine graphene sheet and the adsorbed graphene complex. All calculations employed the DNP (double numerical with $\mathrm{d}$ and $\mathrm{p}$ polarization) basis set, which is comparable to a 6-31G(d,p) Gaussian-type basis set. Geometry optimization calculations used a generalized gradient approximation (GGA) functional that includes exchange-correlation, that is, the Perdew-Burke-Ernzerhof (PBE) functional [16, 17]. PBE-GGA calculations are known to perform well in the description of long-range interactions compared to the local density approximation (LDA) [18]. To describe more accurately the dispersive interactions between graphene and the adsorbed organic compounds, the Tkatchenko-Scheffler (TS) method [19] was employed for dispersion correction. TS is a parameter-free method for the accurate determination of long-range interactions in self-consistent field (SCF) electronic structure calculations. The geometry optimization procedure was performed without any symmetry restrictions. The criteria for both the SCF convergence and the total energy were set to $10^{-6}$ Hartree. The maximum force was converged to 0.002 Hartree per $\AA$, and the maximum displacement parameter was set to $0.005 \AA$. To improve convergence, thermal smearing was used and was set to 0.005 Hartree. To calculate the work function of graphene, the layers were separated by a $20 \AA$ vacuum to minimize interactions between adjacent layers. We used an $8 \times 8 \times 1$ graphene supercell consisting of $128 \mathrm{C}$ atoms.

2.2. Electrostatic Potential Calculations. Electrostatic potential calculations, provided by the $\mathrm{DMol}^{3}$ package, were performed on the periodic structures to obtain the work functions of the pristine graphene sheet and of the graphene doped with amine-based organic dopants. The average potential was calculated along the $c$-direction, which is perpendicular to the graphene surface. The work function $(W)$ was determined using the following equation: $W=V_{\text {vacuum }}-$ $E_{F}$, where $V_{\text {vacuum }}$ is the potential in the vacuum, which is calculated as the average potential between two graphene layers. At large separation from the graphene sheet, the calculated electrostatic potential becomes almost independent of the separation. The calculated electrostatic potential at this distance is defined as $V_{\text {vacuum }}$. $E_{F}$ is the Fermi energy relative to the mean electrostatic potential energy in the graphene layer, and this energy is obtained directly from the electronic state output file generated by $\mathrm{DMol}^{3}$. The work function measures the minimum energy required to separate an electron far from the surface of the graphene and is distinct from the ionization potential because the removed electron is still macroscopically close to the surface and is affected by it. Thus, the vacuum potential is determined as the point at which the calculated average potential becomes flat as distance along the $c$-axis increases; that is, it is the potential at separation from the surface at which the effect on the electron of the surface is at a minimum.

\section{Results and Discussion}

3.1. Molecular Structure Change. DFT geometry optimizations were carried out on both extended pristine graphene (PG) and adsorbed graphene sheets. At first, PBE/DNP calculations with the TS dispersion correction were performed for each organic molecule and for pristine graphene to find the equilibrium geometries of the individual components before optimization of the graphene-dopant complex. Figure 1 shows the chemical structures of each amine-based organic molecule: $\mathbf{1}, \mathbf{2}$, and $\mathbf{3}$. Generally, because of the lone pairs on the nitrogen atoms, amine-based dopants molecules act as electron donors, causing $\mathrm{n}$-type doping of graphene. The benzene rings of the organic dopants can form stable interactions with the graphene sheet due to the formation of strong $\pi-\pi$ interactions. To modulate the electron-donating ability of molecule $\mathbf{1}$, electron-donating alkyl groups (2) and electron-withdrawing acetyl phenyl groups (3) were attached to the benzene rings. To construct the initial input geometry for the graphene-organic complexes, each optimized organic molecule was added to the optimized pristine graphene structure. The benzene rings of the dopants were placed directly above the hexagonal rings of the pristine graphene to maximize the overlap between the carbon atoms. The organic molecules were oriented almost parallel to the graphene sheet with separation of about $\sim 3.26 \AA$, which is characteristic of $\pi-\pi$ stacked systems. Here, for the studied compounds, the chosen separation values were in the range of $2.59-3.67 \AA$ and were determined by averaging reported values [20].

The initial and optimized geometries of the complexes in side-on and top-down views are shown in Figure 2. The optimized structures of the organic molecules in the absence of the graphene sheets show that the organic molecules adopt a nonplanar geometry, mainly to reduce the steric hindrance between ortho-hydrogen atoms in the benzene rings. The butyl groups in 2 extend away from the benzene ring so 
<smiles>CCCCc1ccc(N(CCN(c2ccc(CCCC)cc2)c2ccc(CCCC)cc2)c2ccc(CCCC)cc2)cc1</smiles>

1<smiles>O=C(c1ccccc1)c1ccc(N(CCN(c2ccc(C(=O)c3ccccc3)cc2)c2ccc(C(=O)c3ccccc3)cc2)c2ccc(C(=O)c3ccccc3)cc2)cc1</smiles>

3

FIGURE 1: Amine-based organic molecules. Unmodified phenyl groups (1), alkylphenyl groups (2), and acetylphenyl groups (3). For clarifying the substituent effect, hydrogen atoms at the paraposition to the nitrogen are shown in $\mathbf{1}$.

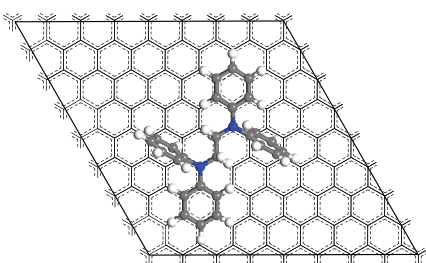

Top view

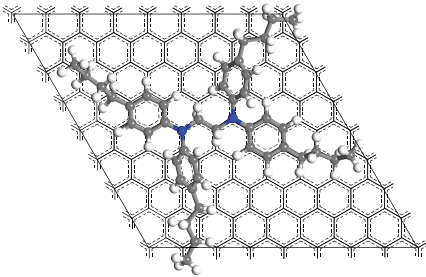

Top view

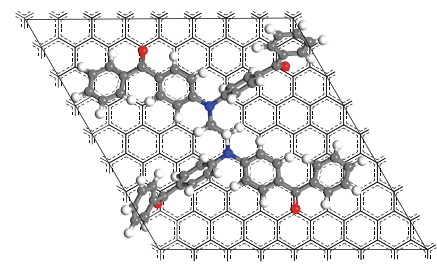

Top view

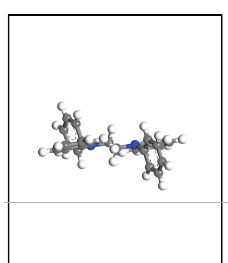

Side view

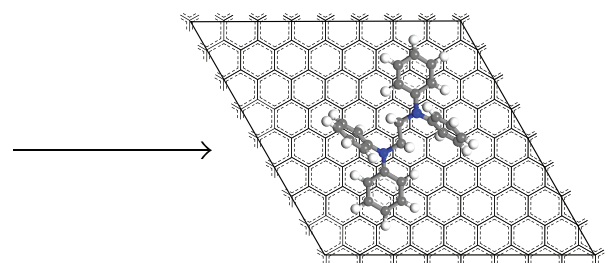

Top view

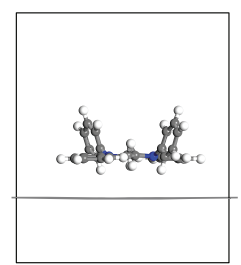

Side view

(a)

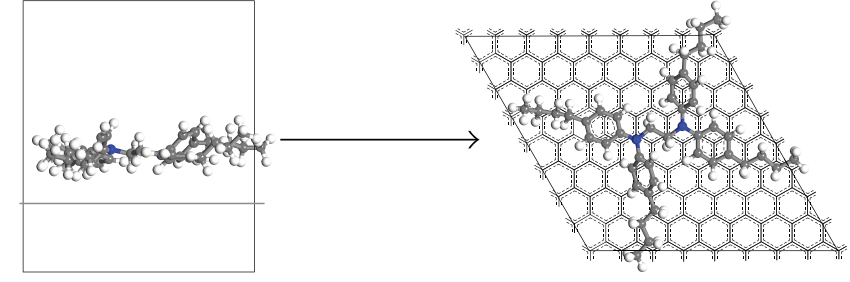

Side view

Top view

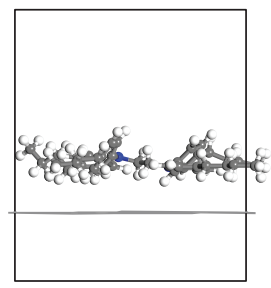

Side view

(b)

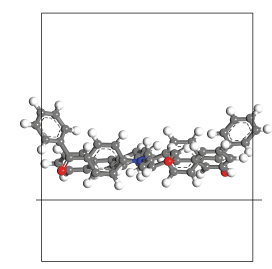

Side view

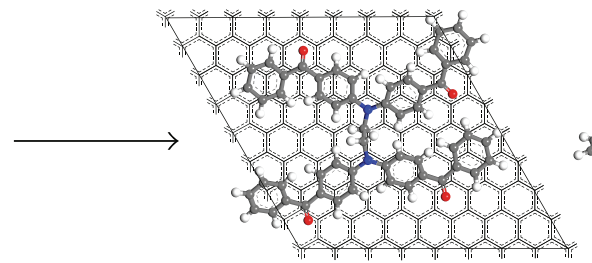

Top view

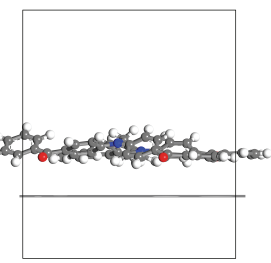

Side view

(c)

Initial geometry

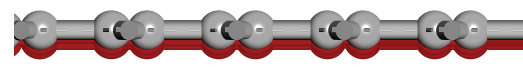

PG+1

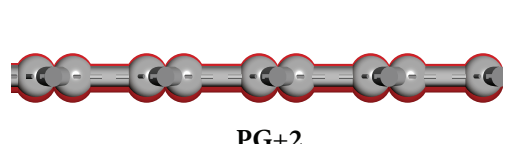

(d)
Optimized geometry

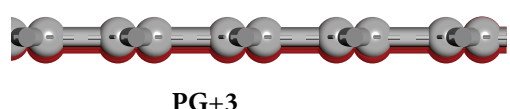

PG+3

Figure 2: Initial and optimized structures of PG and its complexes. (a) $\mathbf{P G}+\mathbf{1}$, (b) $\mathbf{P G}+\mathbf{2}$, and (c) $\mathbf{P G}+\mathbf{3}$. (d) Comparison of the graphene structure before (black) and after (red background) optimization. 
that they do not cause significant steric hindrance. Therefore, $\mathbf{2}$ has a more planar geometry than either $\mathbf{1}$ or $\mathbf{3}$, probably also due to the electron-donating effect of the linear alkyl chains. In 3, the outer benzene rings are displaced out of the plane of the central benzene rings and this may also be due to the steric hindrance between ortho-hydrogen atoms. After structure optimization, $\mathbf{3}$ showed significant structural changes in comparison with the optimized geometries of the other two adsorbed dopants. The distance between the graphene plane and the nearest nitrogen atom in the organic molecules became $3.35 \AA$ for $1,3.38 \AA$ for 2 , and $3.58 \AA$ for 3. Bending of the graphene sheets was observed in the graphene-organic complexes, as shown in Figure 2(d), where the side-on view of the sheets shows the slight bending of the graphene. Most benzene derivatives of organic molecules tend to be located parallel to the graphene layer with an arrangement similar to Bernal's $A B$ stacking configuration [21]. It is possible that this stacking arrangement maximizes the attractive interactions, while minimizing the repulsive interactions between the $\pi$-orbitals of the dopant and graphene. Also, most of the hydrogen atoms of the organic molecule are located in the hexagonal lattice space of the graphene structure, that is, at the centers of hexagons. This could indicate the presence of weak, nonclassical, hydrogen bonding between the electron-rich $\pi$-cloud of graphene and the hydrogen atoms of the organic molecules. This is often termed C-H $\cdots \pi$ hydrogen bonding [20]. This type of hydrogen bonding can also increase the adsorption interaction between the graphene sheets and the adsorbed organic molecules.

The organic molecules also changed their structure after adsorption, as shown in Figure 2. The most significant change was the increase in the proximity of the outer benzene rings of the dopant molecules to the graphene sheet in 3 , which changed from its curved initial optimized geometry to a more planar geometry in the graphene-organic complex. However, the equilibrium distance between graphene and the adsorbed organic dopant is greater in $\mathbf{3}$ than the other two complexes. In addition, the dihedral angles of the ethanediamine linker of the organic molecules $(-\mathrm{N}-\mathrm{C}-\mathrm{C}-\mathrm{N}-)$ were measured to give information on changes in the global structure of the organic molecules when they are adsorbed on graphene. The dihedral angles change from $180.122^{\circ}$ to $189.173^{\circ}, 180.703^{\circ}$ to $184.237^{\circ}$, and $179.416^{\circ}$ to $202.808^{\circ}$ for complexes 1,2 , and 3 , respectively, a total angular change of $+9.051^{\circ},+3.534^{\circ}$, and $+23.392^{\circ}$, respectively, for each compound. All of these structural changes result in the benzene rings becoming closer to the graphene sheet, increasing $\pi-\pi$ interactions through the bending of the backbone structure. The dihedral angle of the ethanediamine linker of compound $\mathbf{3}$ showed the most change because it has the largest area of interaction with the graphene sheet compared to $\mathbf{1}$ and $\mathbf{2}$.

The adsorption energy $\left(\Delta E_{\mathrm{ads}}\right)$ of the organic compounds on the graphene sheet was calculated to evaluate the degree of interaction for each organic compound on the graphene sheet. The adsorption energy was obtained by using the following equation:

$$
\Delta E_{\text {ads }}=E_{\text {doped graphene }}-E_{\text {molecule }}-E_{\text {graphene }},
$$

where $E_{\text {doped graphene, }}, E_{\text {molecule }}$, and $E_{\text {graphene }}$ indicate the total energies of the doped graphene, organic molecule, and graphene, respectively. The adsorption energies of compounds 1,2 , and 3 are $-3.50 \mathrm{eV},-4.14 \mathrm{eV}$, and $-5.79 \mathrm{eV}$, respectively. The adsorption energy increases as the size of the adsorbed molecule increases. The large molecular size of compound 3 gives the largest area of orbital overlap; thus, it can interact more with the confined graphene sheet (i.e., confined to the $8 \times 8 \times 1$ graphene supercell). Also, 3 has twice the number of benzene rings as either 1 or 2 , and these aromatic rings are the main contributor to the interaction between graphene and the adsorbed organic molecule. The molecular size of $\mathbf{2}$ is larger than that of compound 1; therefore, compound $\mathbf{2}$ has larger adsorption energy than $\mathbf{1}$. However, the dihedral angle change of $\mathbf{2}$ is smaller than that of $\mathbf{1}$. This may be due to the alkyl chains in 2 disrupting the interactions between the benzene rings in the organic molecule and graphene; therefore, the benzene rings of $\mathbf{2}$ cannot interact with graphene even though they have increased electron-donating ability compared to 1 . That is, although the alkyl chains increase electron density on the benzene ring, their steric bulk decreases the contact with the graphene sheet. Thus, the observed increase in the adsorption energy for $\mathbf{2}$ is less than we expected based on the electron-donating ability of the alkyl chains. For this reason, it is important to take into account the molecular structure, including steric factors, as well as the electron distribution of the molecule when designing dopants to form stable adsorbates for graphene. Compound $\mathbf{3}$ has more aromatic rings than either $\mathbf{1}$ or $\mathbf{2}$, and the largest dihedral angle change was observed in compound $\mathbf{3}$ after geometry optimization was carried out on the adsorbed complex. From these results, we concluded that the graphene and organic molecules affect the structure of each other on the formation of a physisorbed complex and that the interaction between the two is caused by long-range interactions.

Before we close the discussion of the structural changes that occur on adsorption, we must emphasize that here we have only investigated the physisorption of organic molecules onto graphene. There were almost no structural changes to the graphene sheet after the physisorption of the dopant compared to structural changes to the organic dopants, as shown in Figure 2(d). In calculations where the organic molecule was driven closer to the graphene sheet, one carbon atom in graphene protruded out of the plane and formed a chemical bond with a nitrogen atom of the amine. However, this caused the total energy of the complex to increase, mainly due to the steric hindrance; thus, only the results from equilibrium optimized structures have been discussed in this study.

3.2. Changes to the Electronic Structure. The electronic structure of graphene changes on the adsorption of an organic molecule to graphene [22]. We found that the electronic structure of the doped graphene depended on the electronic properties of the adsorbed organic molecule. The calculated band structures for graphene and the graphene-molecule complexes are shown in Figure 3. Because the Fermi level of pristine graphene exactly crosses the Dirac point, it is well-known that graphene is a zero-gap semiconductor [23]. 


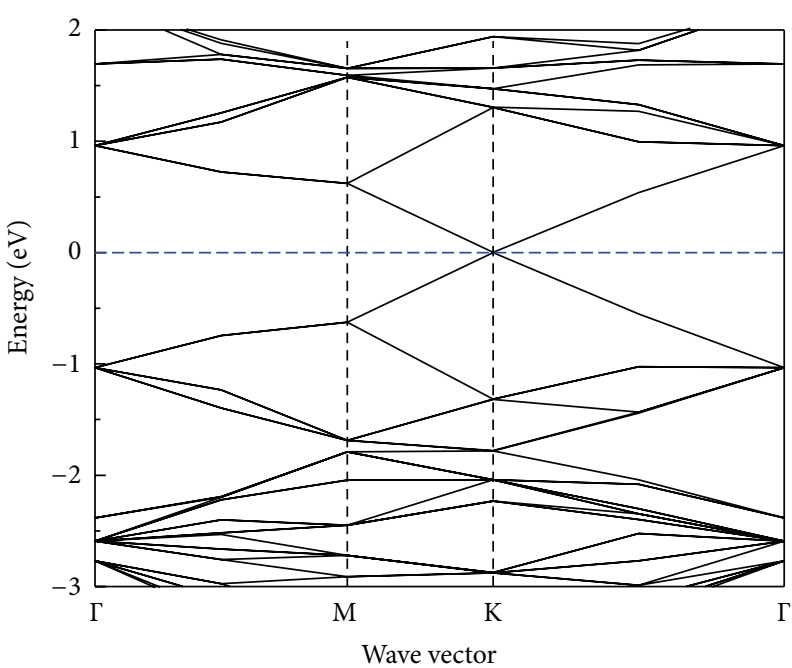

(a)

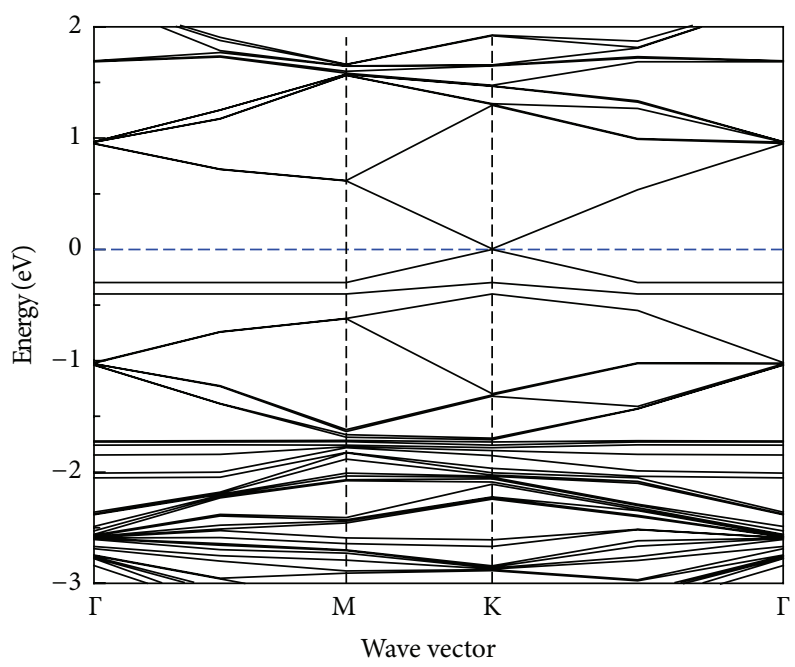

(c)

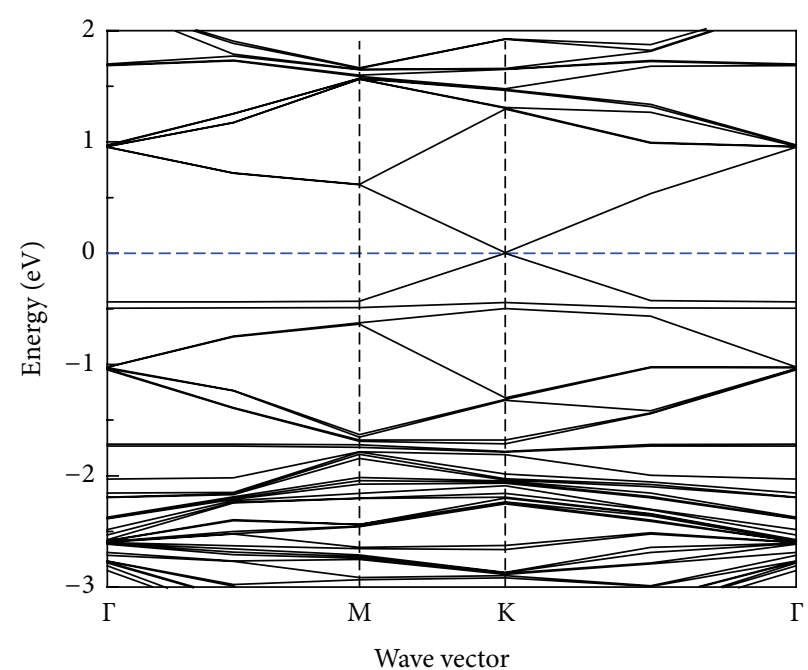

(b)

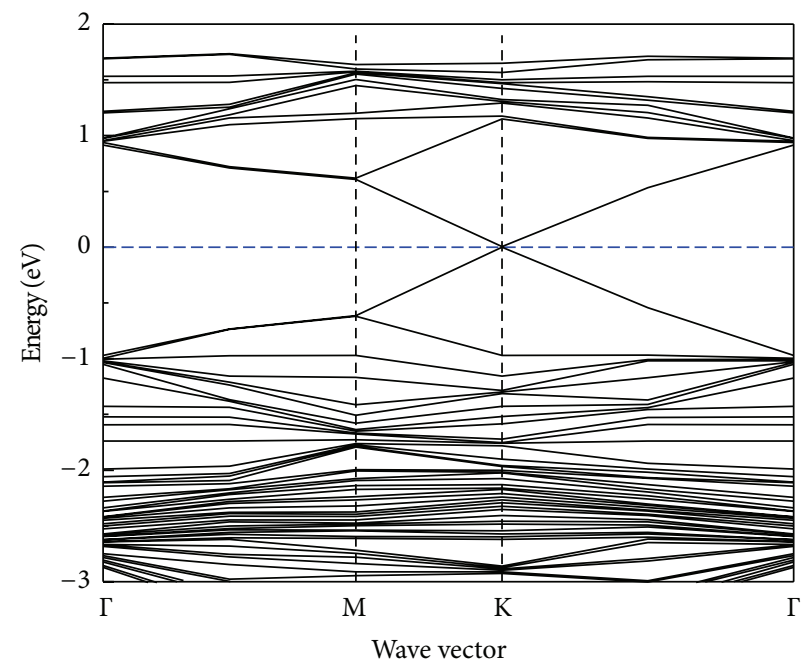

(d)

Figure 3: Electronic band structures in the proximity of the Fermi level of graphene and its complexes. (a) PG, (b) PG+1, (c) PG+2, and (d) PG+3 along high-symmetric points in the Brillouin zone. The energy at the Fermi level $\left(E_{F}\right)$ is shown as a blue dashed line and is set to zero.

Figure 3(a) shows the electronic band structure of pristine graphene along highly symmetric points in the Brillouin zone. The fractional coordinates of these points are $\Gamma(0,0)$, $M(1 / 2,0)$, and $K(1 / 3,1 / 3)$. The energy of the Fermi level $\left(E_{F}\right)$ is set to zero, indicated as a blue dotted line in Figure 3. The valence and conduction bands touch, and the Fermi level bisects two bands at point $K$. Thus, the shift in the band structure at point $K$ gives useful information about changes in the mobility of graphene. As seen in Figures 3(b), 3(c), and 3(d), the electronic band structures of the adsorbed graphene systems show no obvious differences at point $K$ close to the Fermi level compared to pristine graphene; therefore, no changes in mobility should occur after the adsorption of dopants onto graphene. No band structure changes at point $K$ of the Fermi level were observed because no defects are formed during physisorption. For this reason, doping by physisorption is different from other destructive doping techniques such as substitutional doping or the covalent functionalization of graphene [24]. However, when the organic molecules are placed on the graphene sheet, the valence bands become complicated, especially in comparison with the conduction bands. Therefore, we assume that the adsorbed organic molecules have a large impact on the valence band of graphene.

Frontier orbitals and energy diagrams of each adsorbed graphene complex are shown in Figure 4. Analysis of the frontier orbitals of the adsorbed graphene showed that the organic molecules influenced the highest occupied molecular orbital (HOMO) energy of the graphene-molecule complex and the pristine graphene sheet was related to the lowest unoccupied molecular orbital (LUMO) energy of the adsorbed graphene. On formation of the graphene-molecule complex, the HOMO and LUMO energies decreased compared to the energies of the HOMO of the organic molecules 
TABLE 1: HOMO and LUMO orbital energies (in eV) for graphene, molecules, and graphene-molecule systems and Fermi-level energies (in $\mathrm{eV}$ ) for graphene and graphene-molecule systems.

\begin{tabular}{lcccccrr}
\hline & PG & 1 & 2 & 3 & PG+1 & PG+2 & PG+3 \\
\hline HOMO & -5.49 & -4.61 & -4.36 & -5.18 & -4.90 & -4.75 & -5.45 \\
LUMO & -3.49 & -1.37 & -1.19 & -3.06 & -3.51 & -3.59 & -3.57 \\
\hline Fermi level & -4.49 & - & - & - & -4.42 & -4.58 & -4.52 \\
\hline
\end{tabular}

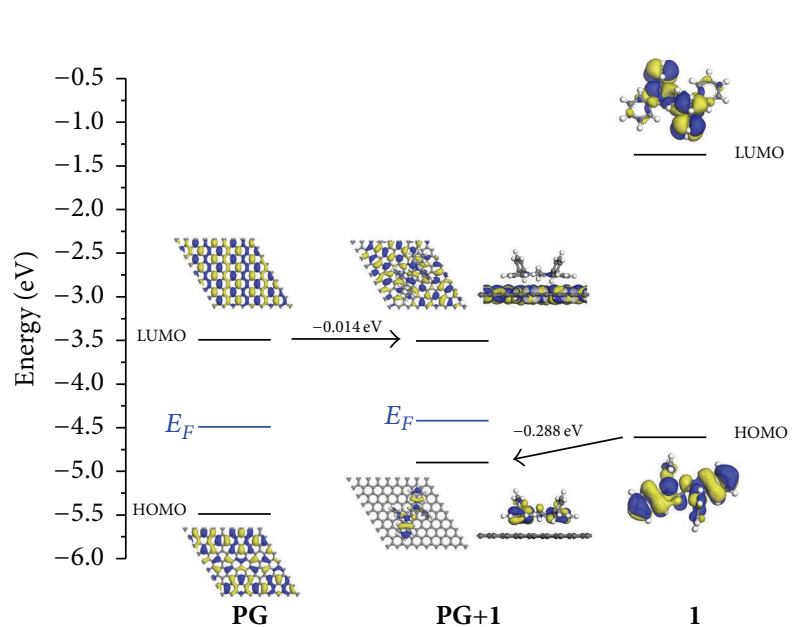

(a)

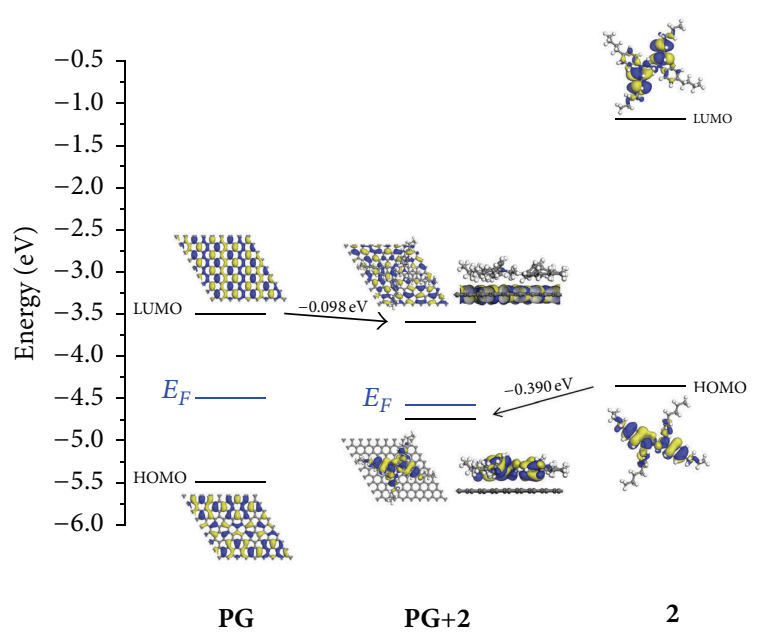

(b)

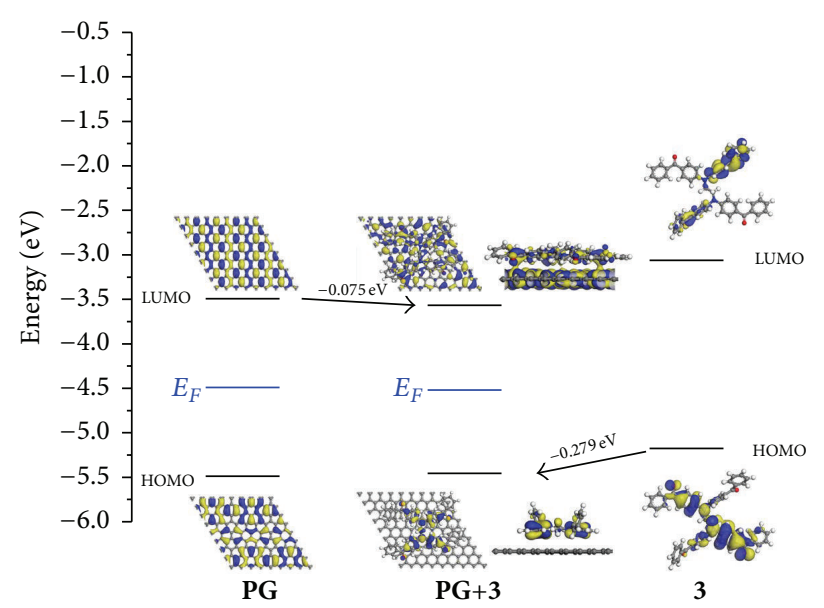

(c)

Figure 4: HOMO and LUMO isosurfaces and energies for graphene complexes. (a) $\mathbf{P G + 1 , ~ ( b ) ~} \mathbf{P G + 2}$, and (c) $\mathbf{P G + 3}$ systems.

and the LUMO of the pristine graphene sheet. Interestingly, when the isovalue for displaying the LUMO isosurface is set equal to 0.01 , the LUMO of 3 is spread over graphene and the organic molecule; in contrast, the LUMOs of $\mathbf{1}$ and $\mathbf{2}$ are localized on the graphene sheet. In frontier molecular orbital theory, the HOMO and the LUMO energies are related to the degree of intermolecular charge transfer. If the HOMO energy of the organic molecules is higher than the Fermi energy level of pristine graphene, charge transfer can take place from the molecule to graphene. The obverse is also true; that is, if the LUMO of the molecule is lower than the Fermi energy level of pristine graphene, charge transfer can occur from graphene to the molecule [25]. The HOMOs and LUMOs of graphene, the organic molecules, and graphene-molecule complexes, and the energies of the Fermi level for both graphene and graphene-molecule systems are summarized in Table 1. The calculated Fermi energy for pristine graphene is $-4.49 \mathrm{eV}$, which is between the HOMO and LUMO energies of graphene. Only the HOMO level of $\mathbf{2}$ is higher than the Fermi level of pristine graphene; therefore, intermolecular charge transfer could occur from 2 to graphene. Intermolecular charge transfer is one of the reasons why the Fermi energy level of the $\mathbf{P G}+\mathbf{2}$ complex is very close to its HOMO energy compared to other adsorbed graphene systems. The Fermi energy level of the graphenemolecule complexes is influenced by the HOMO energy of the adsorbed organic molecules and by the intermolecular charge transfer between the molecule and graphene. Also, 


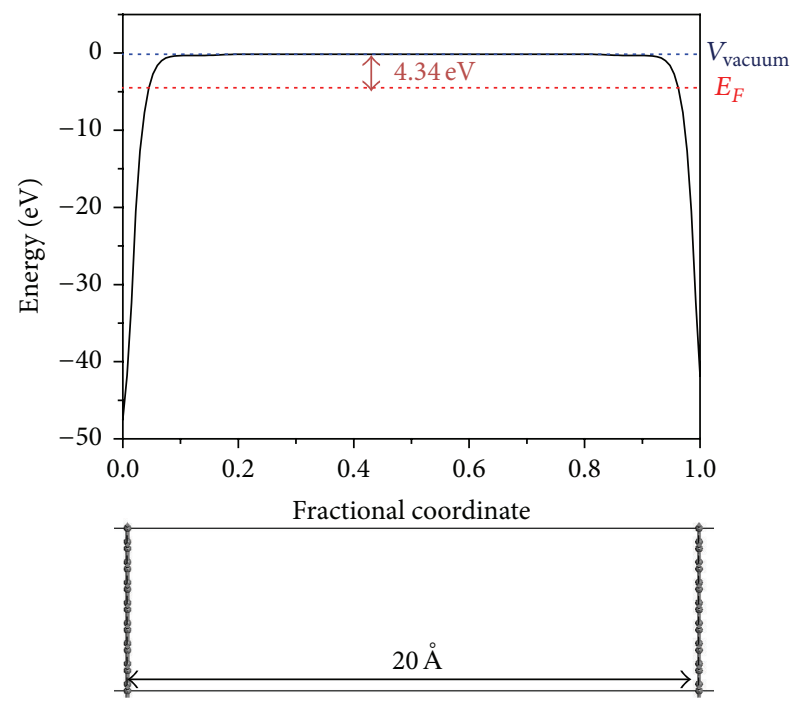

(a)

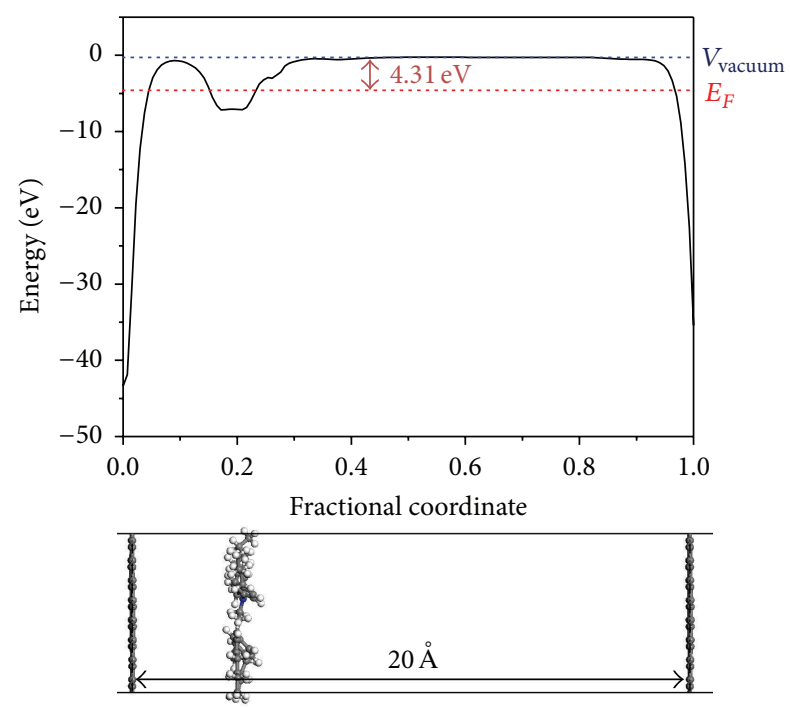

(c)
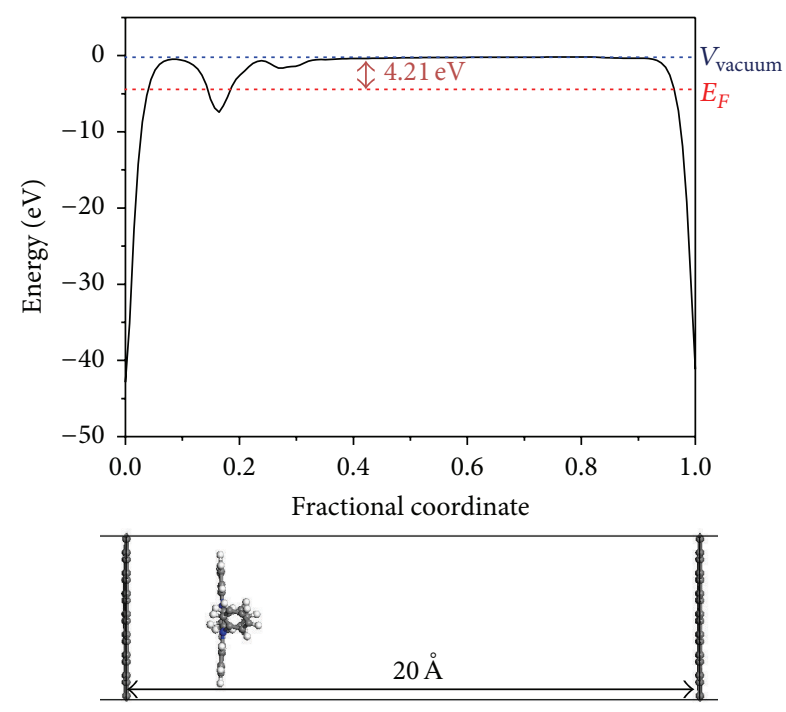

(b)

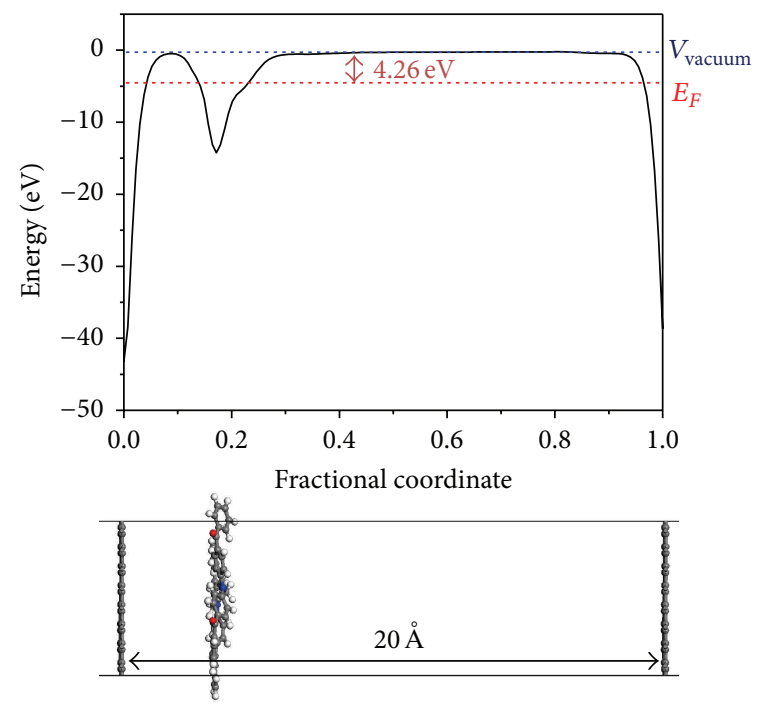

(d)

FIGURE 5: Electrostatic potential diagrams for graphene and the adsorbed complexes. Calculated electrostatic potential diagrams along the c-axis for (a) $\mathbf{P G}$, (b) $\mathbf{P G}+\mathbf{1}$, (c) $\mathbf{P G}+\mathbf{2}$, and (d) $\mathbf{P G}+\mathbf{3}$ systems.

this result is indicative of the electron-donating effect of the alkyl chains on the benzene rings. Increased electron density on the benzene ring may facilitate electron transfer from the adsorbed organic molecules to the graphene sheet.

\subsection{Work Function Calculations. Electrostatic potential cal-} culations were used to probe changes in the work function on adsorption of the dopants onto graphene. Figure 5 shows the electrostatic potential calculations for pristine graphene and for $\mathbf{P G}+\mathbf{1}, \mathbf{P G}+\mathbf{2}$, and $\mathbf{P G}+\mathbf{3}$. Because of the flat average potential between the graphene layers, which are separated by $20 \AA$, there would be no interaction between the graphene layers. A lower electrostatic potential energy is obtained at regions of greater electron density. Because the graphene sheets have a greater electron density than the organic molecules, a lower electrostatic potential energy close proximity to graphene was calculated. At a shift along the $c$ axis of 0.2 (in fractional coordinates), differences were noted in the electrostatic potential profiles that were dependent on the organic dopant; thus, different electrostatic potential diagrams were obtained for each organic compound. The computed vacuum potentials and Fermi energies and work functions are tabulated in Table 2. In a previous report, the work function of CVD-grown monolayer graphene was approximately $4.3 \mathrm{eV}$ [5]. This value corresponds to the work function of our simulations of pristine graphene $(4.34 \mathrm{eV})$; therefore, the DFT electrostatic potential calculation with $\mathrm{DMol}^{3}$ can reproduce experimentally determined work functions quantitatively. The work functions of each grapheneorganic complex are smaller than those of pristine graphene. 
TABle 2: Computed Fermi energies, vacuum potentials, work functions, and the type of doping ( $n$ or $\mathrm{p}$ ) effects of PG and adsorbed graphene sheets.

\begin{tabular}{lcccc}
\hline & $\begin{array}{c}\text { Fermi } \\
\text { energy } \\
(\mathrm{eV})\end{array}$ & $\begin{array}{c}\text { Vacuum } \\
\text { potential }(\mathrm{eV})\end{array}$ & $\begin{array}{c}\text { Work } \\
\text { function }(\mathrm{eV})\end{array}$ & $\begin{array}{c}\text { Doping } \\
\text { effect }\end{array}$ \\
\hline $\mathbf{P G}$ & -4.49 & -0.15 & 4.34 & - \\
$\mathbf{P G}+\mathbf{1}$ & -4.42 & -0.21 & 4.21 & $\mathrm{n}$ \\
$\mathbf{P G}+\mathbf{2}$ & -4.58 & -0.27 & 4.31 & $\mathrm{n}$ \\
$\mathbf{P G}+3$ & -4.52 & -0.26 & 4.26 & $\mathrm{n}$ \\
\hline
\end{tabular}

The work function is influenced by both the Fermi level and the vacuum potential. However, the vacuum potential in work function detection depends on the surface of solid [26]. Because controlling the surface of doped graphene is practically difficult, it is difficult to tune the vacuum potential to a particular level using different organic adsorbates. Therefore, tuning the Fermi energy level, which is possible, is key to altering the properties of graphene by doping. As described in Section 3.2, the HOMO energy level of the organic molecules and the intermolecular charge transfer between molecule and graphene can change the Fermi energy level of the grapheneorganic complex. If we only consider the Fermi energy levels of the graphene-organic molecule complex and pristine graphene, the work functions are in the order $\mathbf{P G}+\mathbf{2}>\mathbf{P G}+\mathbf{3}$ $>\mathbf{P G}>\mathbf{P G}+\mathbf{1}$. However, the presence of the organic molecules on graphene affects the electrostatic environment in the region where the detached electron can escape. As a result, the work functions calculated from our DFT calculations are in the order $\mathbf{P G}>\mathbf{P G}+\mathbf{2}>\mathbf{P G}+\mathbf{3}>\mathbf{P G}+\mathbf{1}$. Interestingly, the energy of the HOMO of organic compounds and of pristine graphene is in the order $\mathbf{2}>\mathbf{1}>\mathbf{3}>\mathbf{P G}$, which tells us that DFT calculation on the isolated molecules cannot predict the work function of graphene-organic complex properly. Thus, the molecular design of organic dopants will benefit from solidstate DFT calculations that simulate the graphene-organic complexes.

Using the DFT calculations, we confirmed that the amine-based organic compounds induce an n-type doping effect on graphene even if there is no change in the band structure at the $K$ point. As a result, when amine-based organic molecules are adsorbed onto the graphene sheet, the adsorbed molecules have no effect on the mobility of graphene. However, the Fermi energy of the grapheneorganic complex is influenced by the different organic molecules.

\section{Conclusion}

To summarize, DFT calculations using PBE/DNP and TS dispersion correction were performed to investigate the effect of doping graphene with amine-based molecules. Dopant molecules with a larger steric size have greater orbital overlap and have a greater binding energy, which stabilizes the adsorbed dopant. Electron transfer from the dopant organic molecules to the graphene sheet was facilitated by increasing the electron density on the benzene rings of the adsorbed molecule. Also, directing some hydrogen atoms of the organic dopant towards the graphene to form nonclassical hydrogen bonds increased the stability of the adsorbed molecules. Work function calculations revealed that the amine-based organic molecules induce an $\mathrm{n}$-doping effect on graphene by decreasing the work function of graphene on formation of the graphene-organic complex. However, there is no change in band structure at the $K$ point of the Fermi level because these systems are doped by physisorption. Therefore, adsorbed amine-based molecules do not affect the mobility of graphene. However, the work function was affected by changing the Fermi level of graphene, and this was observed in the graphene-organic complexes. In conclusion, solid-state DFT calculation is a useful tool to guide the design of organic dopants on graphene.

\section{Conflict of Interests}

The authors declare that there is no conflict of interests regarding the publication of this paper.

\section{Acknowledgments}

This research was supported by the Chung-Ang University Excellent Student Scholarship to Yeun Hee Hwang and by the Public Welfare \& Safety research program through the National Research Foundation of Korea (NRF) funded by the Ministry of Education, Science and Technology (NRF-20100020819).

\section{References}

[1] Z.-S. Wu, W. C. Ren, L. B. Gao et al., "Synthesis of graphene sheets with high electrical conductivity and good thermal stability by hydrogen arc discharge exfoliation," ACS Nano, vol. 3, no. 2, pp. 411-417, 2009.

[2] Y. Xu, Y. Wang, J. Liang et al., "A hybrid material of graphene and poly (3,4-ethyldioxythiophene) with high conductivity, flexibility, and transparency," Nano Research, vol. 2, no. 4, pp. 343-348, 2009.

[3] S. Das Sarma, S. Adam, E. H. Hwang, and E. Rossi, "Electronic transport in two-dimensional graphene," Reviews of Modern Physics, vol. 83, no. 2, pp. 407-470, 2011.

[4] K. S. Kim, Y. Zhao, H. Jang et al., "Large-scale pattern growth of graphene films for stretchable transparent electrodes," Nature, vol. 457, no. 7230, pp. 706-710, 2009.

[5] S. Bae, H. Kim, Y. Lee et al., "Roll-to-roll production of 30-inch graphene films for transparent electrodes," Nature Nanotechnology, vol. 5, no. 8, pp. 574-578, 2010.

[6] H. Ishii, K. Sugiyama, E. Ito, and K. Seki, "Energy level alignment and interfacial electronic structures at organic/metal and organic/organic interfaces," Advanced Materials, vol. 11, no. 8, pp. 605-625, 1999.

[7] L. S. Panchakarla, K. S. Subrahmanyam, S. K. Saha et al., "Synthesis, structure, and properties of boron- and nitrogendoped graphene," Advanced Materials, vol. 21, no. 46, pp. 47264730, 2009.

[8] H. Liu, Y. Liu, and D. Zhu, "Chemical doping of graphene," Journal of Materials Chemistry, vol. 21, no. 10, pp. 3335-3345, 2011. 
[9] D. G. Reuven, H. B. M. Shashikala, S. Mandal, M. N. V. Williams, J. Chaudhary, and X.-Q. Wang, "Supramolecular assembly of DNA on graphene nanoribbons," Journal of Materials Chemistry B, vol. 1, no. 32, pp. 3926-3931, 2013.

[10] X. Dong, D. Fu, W. Fang, Y. Shi, P. Chen, and L.-J. Li, "Doping single-layer graphene with aromatic molecules," Small, vol. 5, no. 12, pp. 1422-1426, 2009.

[11] J. Park, W. H. Lee, S. Huh et al., "Work-function engineering of graphene electrodes by self-assembled monolayers for highperformance organic field-effect transistors," The Journal of Physical Chemistry Letters, vol. 2, no. 8, pp. 841-845, 2011.

[12] Y. Jang, J. H. Cho, D.-H. Kim, Y. D. Park, M. Hwang, and K. Cho, "Effects of the permanent dipoles of self-assembled monolayer-treated insulator surfaces on the field-effect mobility of a pentacene thin-film transistor," Applied Physics Letters, vol. 90, no. 13, Article ID 132104, 2007.

[13] T. Schiros, D. Nordlund, L. Pálová et al., "Connecting dopant bond type with electronic structure in n-doped graphene," Nano Letters, vol. 12, no. 8, pp. 4025-4031, 2012.

[14] S. F. Rastegar, N. L. Hadipour, M. B. Tabar, and H. Soleymanabadi, "DFT studies of acrolein molecule adsorption on pristine and Al-doped graphenes," Journal of Molecular Modeling, vol. 19, no. 9, pp. 3733-3740, 2013.

[15] B. Delley, "From molecules to solids with the $\mathrm{DMol}^{3}$ approach," The Journal of Chemical Physics, vol. 113, no. 18, pp. 7756-7764, 2000.

[16] J. P. Perdew, K. Burke, and M. Ernzerhof, "Generalized gradient approximation made simple," Physical Review Letters, vol. 77, no. 18, pp. 3865-3868, 1996.

[17] J. P. Perdew, K. Burke, and M. Ernzerhof, "Generalized gradient approximation made simple," Physical Review Letters, vol. 77, no. 18, pp. 3865-3868, 1996, Erratum in: Physical Review Letters, vol. 78, p. 1396, 1997.

[18] S. Kurth, J. P. Perdew, and P. Blaha, "Molecular and solid-state tests of density functional approximations: LSD, GGAs, and Meta-GGAs," International Journal of Quantum Chemistry, vol. 75, no. 4-5, pp. 889-909, 1999.

[19] A. Tkatchenko and M. Scheffler, "Accurate molecular van der Waals interactions from ground-state electron density and freeatom reference data," Physical Review Letters, vol. 102, no. 7, Article ID 073005, 2009.

[20] P. P. Zhou and R. Q. Zhang, "Physisorption of benzene derivatives on graphene: critical roles of steric and stereoelectronic effects of the substituent," Physical Chemistry Chemical Physics, vol. 17, no. 18, pp. 12185-12193, 2015.

[21] S. Gowtham, R. H. Scheicher, R. Ahuja, R. Pandey, and S. P. Karna, "Physisorption of nucleobases on graphene: densityfunctional calculations," Physical Review B, vol. 76, no. 3, Article ID 033401, 2007.

[22] O. Leenaerts, B. Partoens, and F. M. Peeters, "Adsorption of small molecules on graphene," Microelectronics Journal, vol. 40, no. 4-5, pp. 860-862, 2009.

[23] J. Yao, Y. Sun, M. Yang, and Y. Duan, "Chemistry, physics and biology of graphene-based nanomaterials: new horizons for sensing, imaging and medicine," Journal of Materials Chemistry, vol. 22, no. 29, pp. 14313-14329, 2012.

[24] D. Wei, Y. Liu, Y. Wang, H. Zhang, L. Huang, and G. Yu, "Synthesis of N-doped graphene by chemical vapor deposition and its electrical properties," Nano Letters, vol. 9, no. 5, pp. 17521758, 2009.
[25] O. Leenaerts, B. Partoens, and F. M. Peeters, "Adsorption of $\mathrm{H}_{2} \mathrm{O}, \mathrm{N} \mathrm{H}_{3}, \mathrm{CO}, \mathrm{NO}_{2}$, and $\mathrm{NO}$ on graphene: a first-principles study," Physical Review B: Condensed Matter and Materials Physics, vol. 77, Article ID 125416, 2008.

[26] R. W. Strayer, W. Mackie, and L. W. Swanson, "Work function measurements by the field emission retarding potential method," Surface Science, vol. 34, no. 2, pp. 225-248, 1973. 

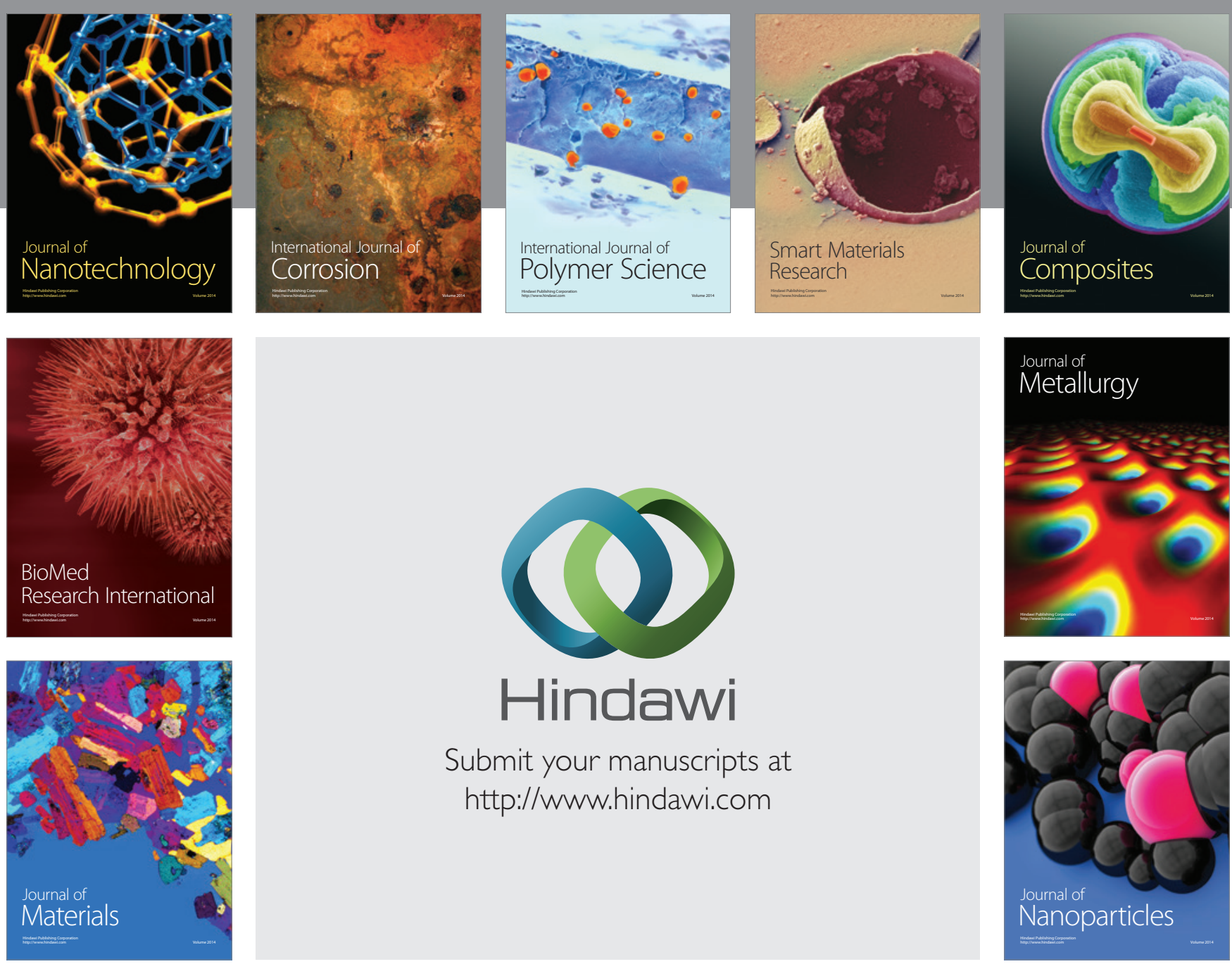

Submit your manuscripts at http://www.hindawi.com
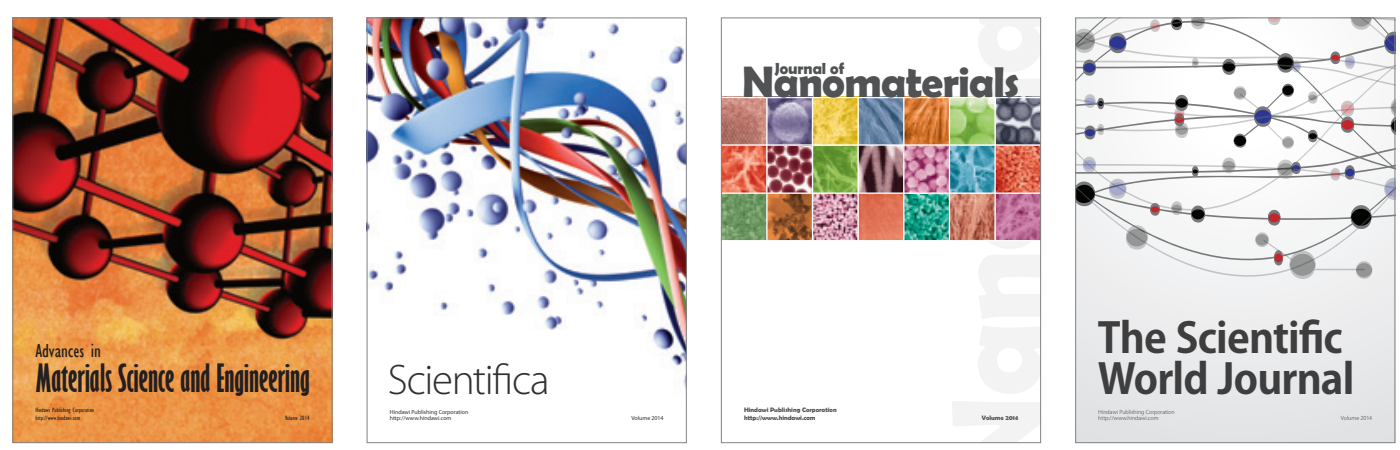

\section{The Scientific World Journal}
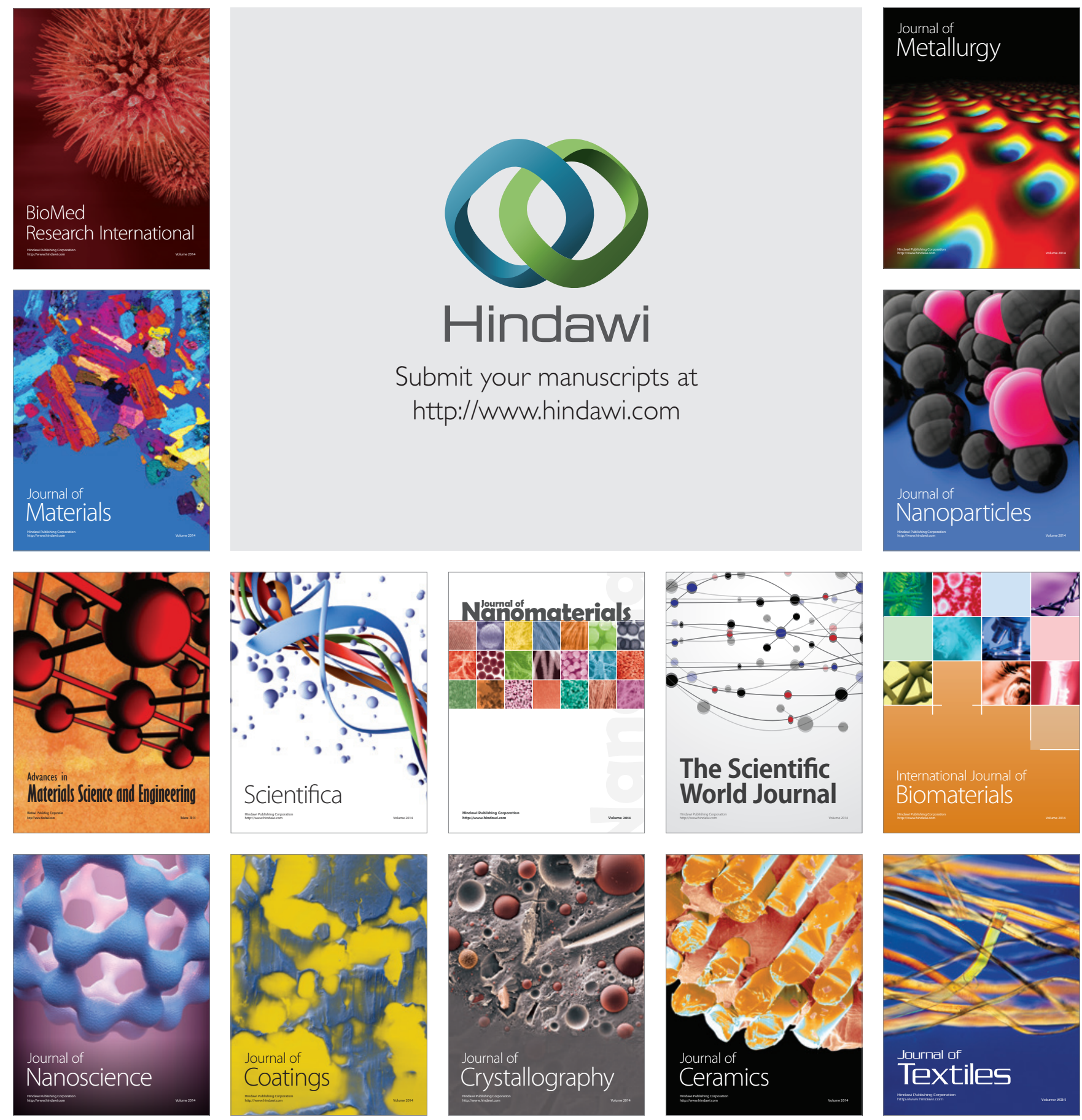